\title{
Different Applications and Techniques for Sentiment Analysis
}

\author{
Suad Alhojely \\ Department of \\ Computer and \\ Information Science \\ Clark Atlanta University, \\ Atlanta, GA 30314,
}

USA

\begin{abstract}
Sentiment Analysis is a continuous field of research in the content mining field. Sentiment Analysis is the computational treatment of feelings, opinions, and subjectivity of content. This study paper handles an extensive review of the last upgrade in this field. Numerous as of late proposed calculations' improvements and different Sentiment Analysis applications are examined and displayed quickly in this review. Sentiment Analysis has as of late assumed a huge part for specialists since examination of online content is helpful for the statistical surveying political issue, business insight, on the web shopping, and logical overview from mental The related fields to Sentiment Analysis that pulled in analysts as of late are talked about. The fundamental focus of this review is to give almost a full picture of Sentiment Analysis is methods and the related fields with brief points of interest. The principle commitments of this paper incorporate the modern classifications of countless articles and the delineation of the late pattern of research in Sentiment Analysis and its related area.
\end{abstract}

\section{General Terms}

Sentiment Analysis.

\section{Keywords}

Opining Mining, Sentiment Analysis, Classification, aspect ranking, techniques

\section{INTRODUCTION}

Sentiment Analysis and Opining Mining speak to the same documented. The main utilization of conclusion investigation by Nasukawa and Yi, 2003[2], and the principal utilization of assessment mining by Dave, Lawrence, and Pennock, 2003 [3]. Already individuals used to get the data from news-paper, magazines, or web articles. Today, the general population share and get data from World Wide Web to catching and imparting their insight. Like this, associations need to study general conclusions for basic leadership. Because of development and progression of informatics empower a ton of ecommerce sites where individuals talk about their sentiment about items and administrations. The purchaser inputs contain valuable and profitable learning for both organizations and clients. Many great items from various merchants have been offered on the web. For instance, Amazon.com registers an aggregate of more than 36 million items. Shoper.com records more than 5 million items from more than 3,000 merchants [13]. Along these lines, this will influence increasingly of open to purchase items on the web, and it will rouse the client to buy online and express sentiments. To purchase online item shopping and give surveys about item or administrations, the quantity of audits becomes quickly. In any case, this site and individuals' surveys are not sorted out which cause trouble for open. These days, the client supposition is basic for social impact. These audits are basic for open since it will help clients settle on the choice effectively and accommodating to the organizations to think about their item's sure and negative trait. Specifically, feeling (assessment) can be defined as supposition communicated by the shoppers. Estimation Analysis speaks to the supposition of the purchaser as positive (like) or negative (aversion) or might be an impartial perspective. The buyer will give careful consideration to the angle from the audits, yet the organization will concentrate on enhancing the feeling about the item. Besides, Sentiment Analysis can be partitioned into three level which are report level, sentence level, and perspective level [14]. Slant investigation strategies are utilized to express audits, supposition, and political issues consequently from the web [15]. Slant Analysis is a branch of Natural Languages Processing to the investigation, inspects, and assesses general feeling by utilizing NLP. This paper clarifies a few methods for feeling investigation and spotlights on conclusion characterization.

\section{BAGKGROUND}

Nasukawa and Yi [2] elucidate that rather than express the finished record into positive or negative, they express opinions associate with positive or negative for a specific subject from an archive. Likewise, they represent the central issue in slant examination which is knowing the notion communicated in writings whether the assumption demonstrates positive or negative assessment. Taylor et al. [4] demonstrate that an outline arrangement of opining digging for tourism which will be advantageous in a lot of enterprises. Additionally, they propose a framework it is ti tackle issue in Lake Distract tourism industry. Haddi, Lui and Shi [5] clarify the opinion of back motion picture eat on the web. They attempt to diminish the nose in the content by utilizing an accumulation of various pre-preparing strategies and utilizing the chi-squared strategy to erase insignificant favorable position that does not affect its introduction. Haddi, Lui and Shi have clarified numerous broad exploratory results. By demonstrating that on two information sets legitimate content pre-preparing exactness fulfilled is like the kind of precision that could be refined in the subject arrangement, it is a less difficult issue. In Moraes, Valiati and Neto [6] focused on looking at amongst SVM and ANN under the state of the prerequisite to accomplished great arrangement exactnesses. Additionally, tests assessed all techniques as a capacity in sack of-words (uni grams) approach specifically terms. Related slant learning writing the vital commitments/discoveries are in two focuses. The principal point is that in term of order precision on a benchmark dataset 
of films audits. The second point is as a finish examination with regards to adjusted information.

\section{LAST PRODUCT ASPECT RANKING}

Chetan Mate in India proposed an item angle positioning structure by utilizing opinion investigation consequently to recognize the principle parts of an uncommon item on online buyer reviews [18]. He proposed work that will base on purchaser audits in four stages. The initial step of the item angle positioning system is that Reviews Extraction and Preprocessing. This progression is basic since it depends on information preprocessing. Opinion investigation connected on unstructured surveys which cause poor execution. Thus, the preprocessing methods to improve result in conclusion investigation are required. Hence, the strategies for preprocessing are stemming and tokenization. Stemming which is to erase the postfix from a word, for example, ing, tion, and so forth. In tokenization, the space from every sentence as appearing in figure 1 will be evacuated. The second step of the item perspective positioning system is that Aspect Identification of the item. There are numerous shopper audits accessible on ecommerce site yet the surveys gathered in various configurations on an alternate site that make an issue. Additionally, some site is a general evaluating on any item like CNet.com, another site the surveys are in section in configuration content like Viewpoints.com. $\mathrm{Hu}$ and Liu proposed an approach that recognizes the thing and a thing expression. The thing and thing expression are numbered then the just fitting thing is spared as the angle [16]. The third step of the item viewpoint positioning structure is Sentiment Classifier. Estimation investigation is the kind of Natural Languages Processing which utilized as a part of following extremity of individuals about item surveys. Opinion investigation orders a content as positive, negative, or impartial. There are two sorts of arrangement strategies learning: directed learning and unsupervised learning. The dictionary based methodologies are unsupervised which utilize it will get slant extremity on every part of word and expression [17]. Then again, the directed learning relies on upon the preparation dataset. Additionally, he specifies to numerous learning based arrangement show like SVM, Naïve Bayes, and Maximum Entropy (ME). The forward stride of item viewpoint positioning structure is Aspect Ranking Algorithm. The general sentiment in the audits of the item is a sum of supposition given to particular perspectives in that survey. In this way, the assumptions on vital or insignificant angles have solid and powerless effects of the general supposition. Their proposed structure utilizes AFINN lexicon approach, which comprises of numerous English words evaluated valence with a whole number between (-5) and $(+5)$ as positive and negative. They endeavor to make an angle positioning calculation to recognize the vital viewpoints.

\section{INSTURMENTS OF SENTIMENT ANALYSIS CLASSIFITION}

There are such a variety of open-source content examination devices utilized for characteristic dialect preparing, for example, data extraction, and arrangement can likewise be connected to estimation investigation. The accompanying is instruments utilized for Sentiment Classification:

1. 1-NTLK: A characteristic dialect toolbox is a device for content preparing, indexing, tokenization, ceasing, labeling, parsing, and so on. It gives simple to-utilize interfaces to additional than 50 corpora and lexical assets, for example, Word Net, alongside a suite of http://www.nltk.org/

2. Door: Beneficial on the off chance that you need to make a pipeline. Dialect investigation modules for designers contribute different dialects are accessible to be connected to your pipeline.

3. OpenNLP: play out the most widely recognized NLP assignments, for example, POS labeling, named substance extraction, lumping, what's more, co-reference determination.

http://opennlp.apache.org/StanfordCoreNLP: If you require grammatical form classes, syntactic investigation (express structure or reliance examination), co-reference or named elements in content.

4. OpinionFinder: It backings to distinguish singular sentences and to case distinctive parts of subjectivity in these sentences, including the conclusion holder of the subjectivity and words that are joined into expressions imparting positive or negative suppositions. http://code.google.com/p/opinionfinder/

5. Ling Pipe: Ling Pipe is utilized for etymological preparing of content including, bunching indexing and element extraction, and so on http://nom de plume i.com/lingpipe/.

\section{CLASSIFICATIONS}

Assumption groupings depend on an extremity, which may get to be sure, negative, or unbiased. That is mean suppositions might be arranged into positive, negative, or unbiased. Also, there is a forward sort which is a useful conclusion which acquires proposal to improve the item [7]. Sentiments are grouped into three classes: the first is immediate conclusions which supposition holder specifically assault to target. The second one of assessment is similar suppositions which are feeling holder think about among element. The third one is backhanded conclusions, which are inferred as in figures of speech or communicated reversed as in mockery. Specialists have concentrated on estimation examination into three level:

\subsection{Document Level Sentiment Classification}

Archive level notion order plans to group the whole report as positive or negative. There are many genuine works utilize one of the two sorts of grouping procedures which are a Supervised strategy and Unsupervised technique to manufacture level report estimation.

\subsubsection{Supervised technique:}

Opinion arrangement is performed at record level feeling [1]. Assumption arrangement can be utilized as a regulated order issue with four classes positive, negative, impartial, and valuable [8] [9] [10] [11]. Likewise, regulated demand machine-learning calculations like SVM Support Vector Machines to close the connections between the feelings that communicated and content fragment. A considerable measure of analysts found that regulated learning systems can perform well in SVM and Naïve Bayes (Pang et al. 2008) [8].

\subsubsection{Unsupervised technique:}

Unsupervised characterization is performed at the sentence level [1]. There are two sorts of unsupervised order, which are vocabulary based, and syntactic-design based. Sentence and angle level conclusion order for the dictionary based can be utilized. 


\subsection{Sentence Level Sentiment Classification}

In this level, the assignment is to decide every sentence in the archive as positive or negative assessments. Sentence level notion examination has grouped the extremity. This level is near archive level yet here it fulfilled by each sentence [12]. Be that as it may, there might be unpredictable sentences in the content which make the sentence level is not useful. There are two stages in level sentence conclusion done in each and every sentence: to start with, every sentence grouped, as subjective or objective, and the second one is the extremity of subjective sentence are closed.

\subsection{Aspect Level Sentiment Classification}

It assumes that a record has a hold sentiment on numerous elements and their angles. Viewpoint level grouping needs disclosure of these elements, angles, and feelings for each of them.

\section{SENTIMENT ANALYSIS CLASSIFICATION TECHNIQUES}

Sentiment Analysis Classification Techniques partitioned to three methods, which are Machine Learning (ML) approach, Dictionary based approach, and Hybrid approach. The ML approach stratifies the ML calculations and utilizations etymological advantage. The order techniques that utilization ML approach can be separated into Supervised and Unsupervised learning strategy. The Lexicon-construct approach depends in light of supposition vocabulary [19]. Dictionary considers as an imperative pointer for supposition, which called conclusion word. Vocabulary isolated into word reference based approach and corpus approach. The lexicon based approach discovers sentiment seed words, and afterward seek the word reference of their antonyms or equivalent words. Be that as it may, the corpus-based approach begins with a rundown of seed feeling and at that point finds another sentiment in a major corpus to attempt help finding sentiment words in setting. The corpus approach utilizes the factual or semantic technique to determinate the extremity of opinion. The Hybrid approach is a blend of ML approach and Lexicon-based approach, and it is extremely famous in Lexicon-based approach as appeared in figure2.

\section{RESEARCH ISSUES}

There are numerous open research issues in assumption investigation, including:

1. There is a requirement for better demonstrating of compositional estimation. At the sentence level, this implies more exact estimation of the general sentence opinion of the assessment bearing words, the supposition shifters, and the sentence structure.

2. Every item has numerous names that allude to it even inside similar archive and unmistakably crosswise over reports. This issue of programmed substance determination is not yet tackled. Another related significant obstacle is treatment of anaphora determination in an exact way. This is an issue for angle extraction as well, that is, the manner by which to gathering viewpoints, for instance, "battery life" and "power utilization" allude to similar part of a telephone.

3. At the point when a record examines a few substances, it is essential to distinguish the content important to every element. Current precision in distinguishing the pertinent content is a long way from attractive.
4. In spite of the fact that there are some methodologies that utilization characterization techniques to recognize mockery, they are not yet incorporated inside self-ruling supposition examination frameworks.

5. Loud messages (those with spelling/linguistic slip-ups, missing/risky accentuation and slang) are still a major test to most assessment investigation frameworks.

6. A significant number of the announcements about elements are truthful in nature but then despite everything they convey opinion. Current assessment examination approaches decide the assumption of subjective proclamations and ignore such target explanations. There is a requirement for calculations that utilization setting to append notion scores to objective (accurate) articulations. Such proclamations happen every now and again in news articles.

\section{ECONOMIC IMPACT OF REVIEWS}

Numerous readers of online audits say that these surveys fundamentally impact their buying choices [20]. In any case, while these readers may have trusted that they were "fundamentally impacted," recognition and reality can vary. A key motivation to comprehend the genuine monetary effect of surveys is that the aftereffects of such an investigation have important ramifications for how much exertion organizations may or ought to need to exhaust on online notoriety checking and administration. Given the ascent of online trade, it is not amazing that an assemblage of work focused on the financial aspects, what's more, showcasing writing considers the subject of whether the extremity (regularly alluded to as "valence") as well as the volume of surveys accessible online affect, good shopper pursuing. Financial specialists have taken a gander at the estimation of keeping up a decent notoriety as a way to beat these issues [21, 22, 23, 24], among different systems. One approach to secure a decent reputation is, of course, by getting numerous positive audits of oneself as a shipper; another is for the items one offers to get multiple positive reviews. For the reasons for our discourse, we respect tests wherein the purchasing is speculative as being out of extension; rather, we concentrate on financial investigations of the conduct of individuals occupied with genuine shopping and spending real money.

\section{ACKNOWLEDGEMENTS}

The author would like to think Dr. Roy George for his comments and suggestions during prepare manuscript. Saudi Arabian Cultural Missions supports the author financially. This support is thankfully appreciated.

\section{REFERENCES}

[1] Goutam Chakraborty, Murali Pagolu, Satish Garla, "Text Mining and Analysis: Practical Methods, Examples, and Case Studies Using SAS” Pp 181-184, 2013.

[2] T. Nasukawa, "Sentiment Analysis: Capturing Favorability Using Natural Language Processing Definition of Sentiment Expressions," pp. 70-77, 2003.

[3] K. Dave, I. Way, S. Lawrence, and D. M. Pennock, "Mining the Peanut Gallery: Opinion Extraction and Semantic Classification of Product Reviews,” 2003.

[4] E. Marrese-Taylor, J. D. Velasquez, and F. BravoMarquez, "Opinion Zoom: A Modular Tool to Explore Tourism Opinions on the Web," 2013 IEEE/WIC/ACM Int. Jt. Conf. Web Intell. Intell. Agent Technol., pp. 261- 
264, Nov. 2013.

[5] E. Haddi, X. Liu, and Y. Shi, "The Role of Text Preprocessing in Sentiment Analysis," Procedia Comput. Sci., vol. 17, pp. 26-32, Jan. 2013.

[6] R. Moraes, J. F. Valiati, and W. P. Gavião Neto, "Document-level sentiment classification: An empirical comparison between SVM and ANN," Expert Syst. Appl., vol. 40, no. 2, pp. 621-633, Feb. 2013.

[7] R. Arora and S. Srinivasa, "A Faceted Characterization of the Opinion Mining Landscape," pp. 1-6, 2014.

[8] B. Pang and L. Lee, "Opinion Mining and Sentiment Analysis," Found. Trends ${ }^{\circledR}$ Inf. Retr., vol. 2, no. 1-2, pp. $1-135,2008$.

[9] B. Pang, L. Lee, and S. Vaithyanathan, "Thumbs up? sentiment classification using machine learning techniques," presented at the Proceedings of the ACL-02 conferenceon Empirical methods in natural language processing - Volume 10, 2002.

[10] D. Bespalov, B. Bai, A. Shokoufandeh, and Y. Qi, "Sentiment Classi fi cation Based on Supervised Latent n-gram Analysis," pp. 375-382, 2011.

[11] M. Gamon, "Sentiment classification on customer feedback data: noisy data, large feature vectors, and the role of linguistic analysis."

[12] H. Yu and V. Hatzivassiloglou. "Towards answering opinion questions: separating facts from opinions and identifying the polarity of opinion sentences. In Proceedings of the 2003 conference on Empirical methods in natural language processing, EMNLP '03, pages $129-136$.

[13] Zheng-Jun Zha, Jianxing Yu, Jinhui Tang, Meng Wang, Tat-Seng Chua," Product Aspect Ranking and Its Applications". IEEE2014.
[14] Bing Liu, "Sentiment Analysis and Opinion Mining" pp.7-140,2012.

[15] Anand Mahendran and Anjali Duraiswany, "Opinion Mining for text classification", International Journal of Scientific Engineering and Technology (2277-1581), Vol No.2,2013.

[16] M. Hu and B. Liu, "Mining and summarizing customer reviews," in Proc. SIGKDD, Seattle, WA, USA, 2004, pp. 168-177.

[17] Bing, Liu "sentiment analysis and opining mining" pp 7140,2012

[18] Chetan Mate "Product Aspect Ranking using Sentiment Analysis: A Survey "Vol No 1,2015.

[19] Suad Alhojely. Sentiment Analysis and Opinion Mining: A Survey. Internationl Journal of Computer Application 150(6):22-25, September 2016.

[20] comScore/the Kelsey group. Online consumergenerated reviews have a significant impact on offline purchase behavior. Press Release, November 2007.

[21] Douglas W. Diamond. Reputation acquisition in debt markets. Journal of Political Economy, 97(4): 828-862, 1989.

[22] Benjamin Klein and Keith Leffler. The role of market forces in assuring contractual performance. Journal of Political Economy, 89(4):615-641, 1981.

[23] Carl Shapiro. Consumer information, product quality, and seller reputation. Bell Journal of Economics, 13(1):20-35, 1982

[24] Carl Shapiro. Premiums for high quality products as returns to reputations. Quarterly Journal of Economics, 98(4):659-680, 1983.

\section{APPENDEX}

Consumer Review Processing
Aspect Identification Sentiment Classification

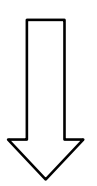

Aspect Evaluation
Overall Rating

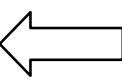




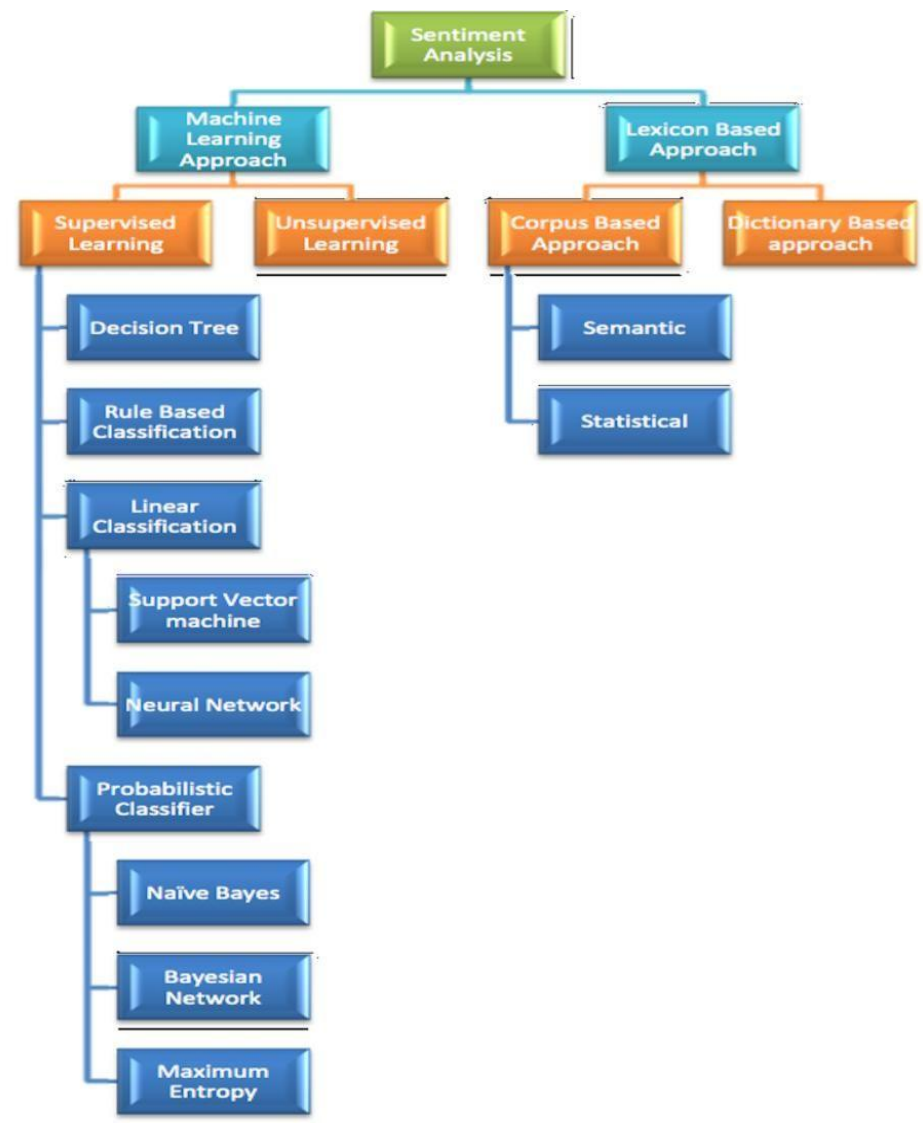

Figure2: Sentiment Analysis Classification Technique 